\title{
JUVENILE DERMATOMYOSITIS
}

\author{
Sigrid Covaci, ${ }^{1,2}$, Oana-Maria Farkas², Alexis-Virgil Cochino, ${ }^{1,2}$ \\ 1 "Carol Davila" University of Medicine and Pharmacy, Bucharest \\ 2 "Alessandrescu-Rusescu” National Institute for Mother and Child Health, Bucharest
}

\begin{abstract}
Juvenile dermatomyositis is a severe multisystemic autoimmune disease of uncertain origin, based on chronic perivascular inflammation, involving mainly the skin and the muscles. We present a dermatomyositis case with classical skin involvement, where muscle damage occurred more than a year after disease onset.

Keywords: juvenile dermatomyositis, dermatomyositis sine myositis, muscle weakness, myositis, heliotrope rash, Gottron sign
\end{abstract}

\section{INTRODUCTION}

Pediatric immunological disorders, although a "fashionable" topic for online social media, are often diagnosed late, being the source of avoidable complications/sequelae. One of the most frequently encountered - juvenile dermatomyositis (JDM) may vary from the simplest and mildest forms (such as dermatomyositis without myositis), through the classic ones (skin and muscle involvement), and up to severe forms with threatening events (with neurological or hematologic complications). As a gold standard test does not exist, diagnosis is determined by a set of criteria (Bohan and Peter):

1. symmetrical proximal muscle weakness: pelvic and scapular girdles

2. increased muscle enzymes: $\mathrm{CPK}, \mathrm{LDH}$, AST, aldolase

3. electromyographic changes (EMG): polyphasic short small motor unit potential, fibrillations, positive sharp waves, increased insertional irritability; bizarre, high frequency, repetitive discharges

4. muscle biopsy: fiber size variation, particularly perifascicular atrophy, degeneration/ regeneration; mononuclear inflammatory infiltrate, most often perivascular

5. skin manifestations: heliotrope rash, erythematous papules (Gottron papules) on the extensor surfaces of the metacarpophalangeal joints (MCP), proximal interphalangeal (PIF), elbows and knees.

JDM requires the presence of rash and other 3 criteria (definite diagnosis), rash and 2 criteria (probable diagnosis) or rash and 1 criterion (possible diagnosis).

Disease onset is usually insidious, with general signs (irritability, fatigue, low/high grade fever, anorexia, weight loss) that may precede the diagnosis by 3-6 months (1).

Skin involvement may be the first manifestation of the disease (preceding the occurrence of muscle damage with a time of months to years). There is no correlation between the degree of skin and muscle involvements (2).

Characteristic lesions consist of heliotrope rash (periorbital purplish color which may be accompanied by edema of the eyelids) and Gottron papule/ signs (erythematous papules on the extensor surfaces of the MCP, PIF, elbows, knees, external malleolus). Another skin sign, less characteristic, is the facial rash with malar distribution. Sometimes changes can be seen around the nails - hyperemic nail bed because of dilated capillary loops (which sometimes can be seen with the naked eye) or ragged cuticles (3). Muscle damage is symmetric and predominate in the girdles, especially the pelvic one (4). Neck flexor muscles damage is possible, as well as the stomach involvement. Visceral 
manifestations (gastrointestinal, lung, heart) indicate severe disease. Particular attention should be paid to the occurrence of dysphagia (which may suggest esophageal hypomotility), dysphonia and nasal voice.

Specific or myositis associated autoantibodies are not required for the diagnosis; their utility resides in defining disease subtypes and possibly as prognostic factors.

A case of JDM is presented, covering half of the possible evolving spectrum of the disease, from dermatomyositis without myositis, to complete JDM.

\section{CASE PRESENTATION}

A 3 years and 1 month old female patient presented in January 2014 for determination of diagnosis, with unremarcable personal and family history. Disease onset was in April 2013, with erythematous eruption of the face (respecting the forehead and perioral area) after sun exposure (about 2 hours); other areas exposed to the sun (hands) were unaffected.

A dermatology clinic visit established an allergodermia/sunburn diagnose and antihistamine treatment was recommended, along with sunscreens. During the summer, the rash persists, waxing and vaning, but new erhytematous elements occur over the elbows, knees, PIF, MCP and the back of the neck, for which she only receives anti-allergy treatments (with no effect). The first set of blood work $\left(15^{\text {th }}\right.$ of October 2013) were within normal limits (complete blood count, transaminases, ESR), except for a mild anaemia ( $\mathrm{Hgb} 9.4 \mathrm{~g} / \mathrm{dl})$. Up to the initial presentation patient never presented muscle weakness, weight loss, hoarseness, dysphagia or dyspnoea. Significant clinical elements from the first consultation were:

- facial rash respecting the forehead and perioral area (Fig. 1)

- bilateral ear hyperaemia (Fig. 1)

- Gottron signs (Fig. 2, 3, 4)

- linear erythema on extensor surfaces of the thumbs

- systolic heart murmur grade II-III/VI

- no dysphagia/dysphonia; normal muscle force ("she playes, rides bicycles, go up and down stairs, the same as other children")

- negative Gowers' maneuver

- no abdominal pain, melaena or haematemesis

Blood work revealed hypochromic microcytic anemia, mild thrombocytopenia, absent inflamma-

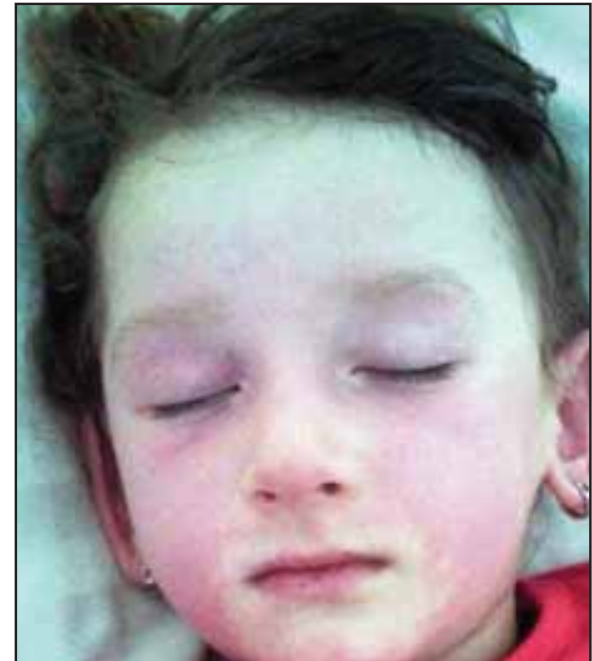

FIGURE 1. Facial rash, respecting the forehead and perioral area

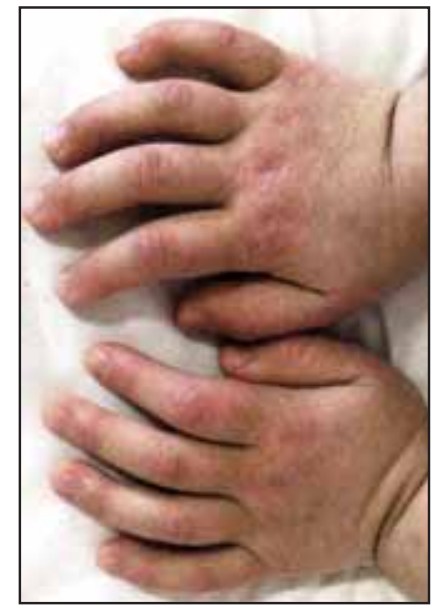

FIGURE 2. Gottron papules over MCP, PIF

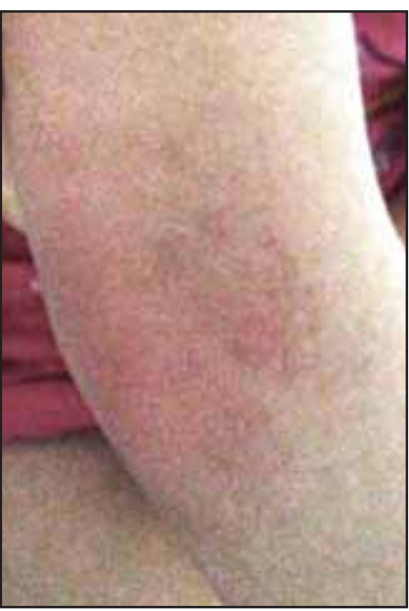

FIGURE 3. Erhytematous plaques over elbows

tory markers, transaminases and muscle enzymes with normal values. Nail fold capilaroscopy depicts branched capillary loops (Fig. 5), dilated capillaries - some becoming megacapilare - and decreased capillary density (Fig. 6). Given the rash characteristics and the possibility of delayed muscle damage in JDM (months or years), a diagnosis of dermatomyositis sine myositis was considered (dermato- 


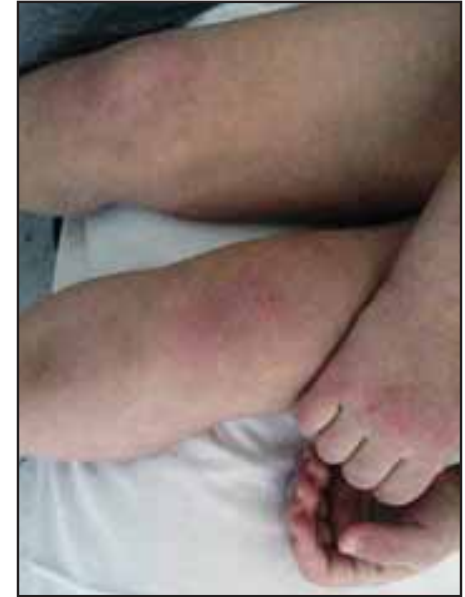

FIGURE 4.

Erhytematous plaques over knees

myositis without myositis). Myositis autoantibody panel found positive PL-7 antibodies (associated with arthritis risk, interstitial pulmonary fibrosis and mild myositis). Treatment with prednisone was initiated at a dose of $5 \mathrm{mg} /$ day.

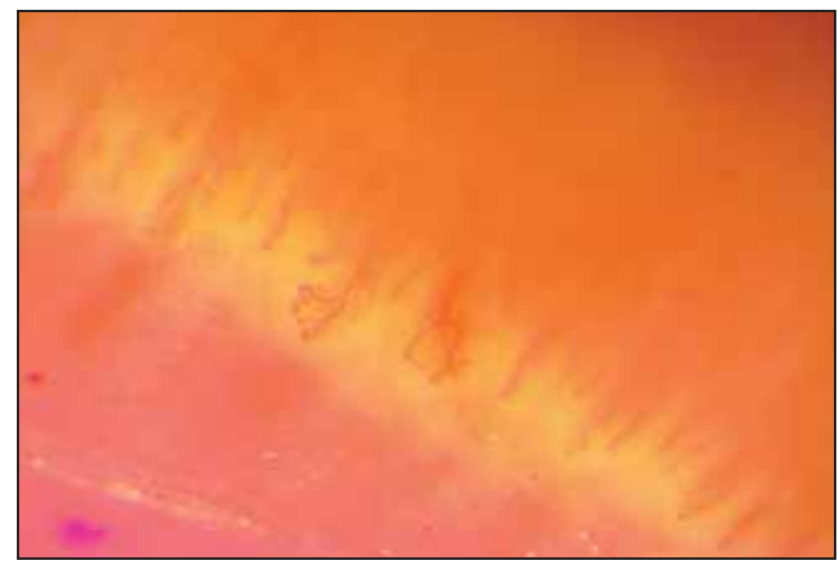

FIGURE 5. Nail fold capilaroscopy - capillary loss, ramified capillaries

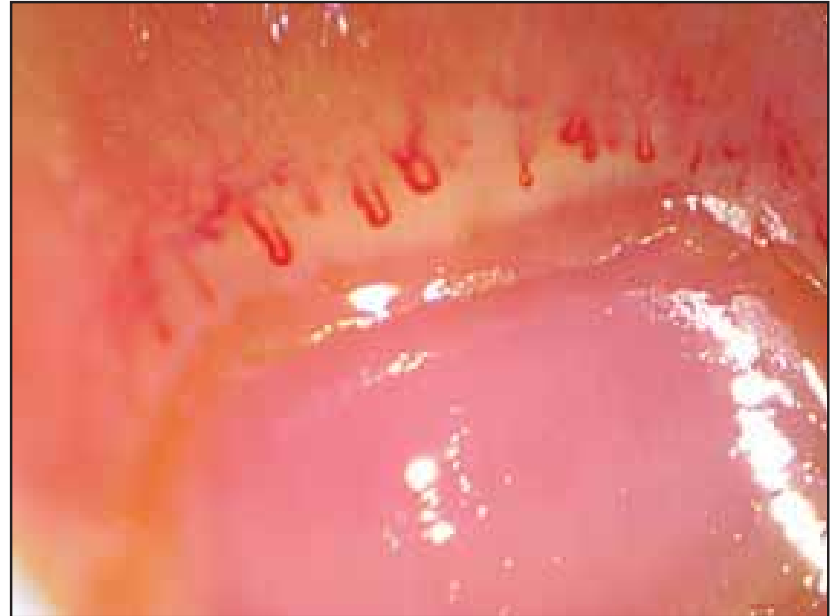

FIGURE 6. Nail fold capilaroscopy - capillary loss, enlarged and giant capillaries

Under this treatment evolution was favorable, alleviating Gottron signs and heliotrope facial rash until May 2014 when, still in the absence of objective signs of muscle injury, chemical rhabdomyolisis appears (elevated AST, CPK, LDH), which determines the increase of prednisone dose to $10 \mathrm{mg} /$ day $(=0.6 \mathrm{mg} / \mathrm{kg} /$ day $)$.

After two more months (July 2014) the patient presents multiple sites arthritis, worsening rash with discrete vasculitis pattern (reticular, fading under pressure), and muscle weakness. She maintains elevated LDH but normal CPK, AST. Prednisone dose increase to $15 \mathrm{mg} /$ week and Methotrexate (MTX) treatment initiation at a dose of $5 \mathrm{mg} /$ week was decided, with favorable evolution, both

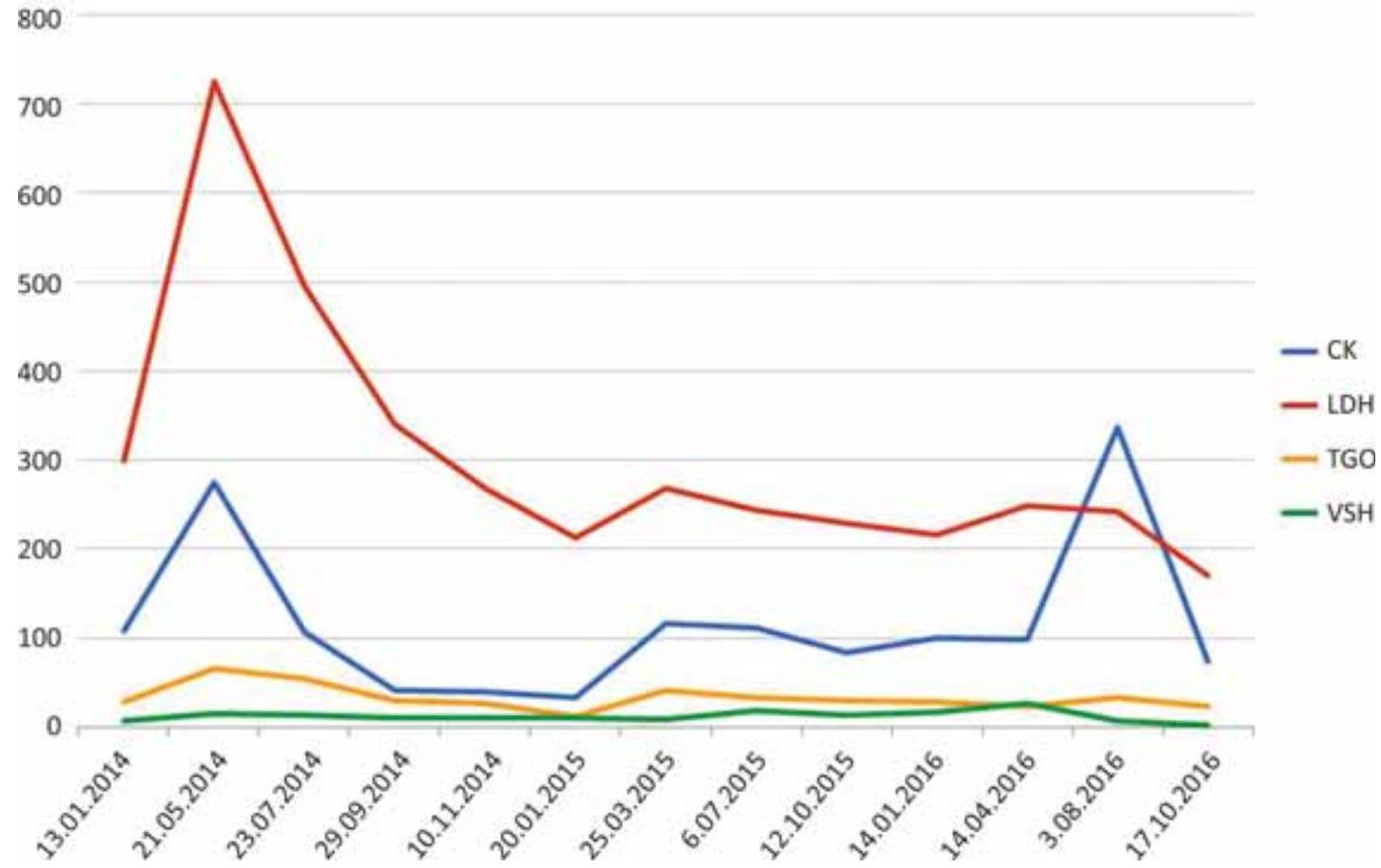

FIGURE 7. Muscle enzymes and ESR evolution 
clinical (muscle and skin) and biological (LDH continued to fall, normal blood works in rest).

Given the relatively high dose of prednisone ( $\sim 0.9 \mathrm{mg} / \mathrm{kg} /$ day) needed to achieve this, with unacceptable long term side effects, it was decided to further increase methotrexate dose in an attempt to provide adequate control of the disease and wean the steroids (suspended in May 2015). In August 2016, without worsening of clinical signs, mild rhabdomyoslisis occurs again; to return the disease under control MTX dose is slightly increased and low dose prednisone is reintroduced. At the time of this paper writing, the disease is controlled both clinically (no rash; normal muscle strength) and biological (muscle enzymes within normal range).

\section{DISCUSSIONS}

Our case highlights the importance of early diagnosis, close monitoring (even in mild cases with only skin involvement) and prompt treatment tuning, following disease activity (time-varying). The fulfillment of these conditions result in adequate control of the disease and prevents its complications (e.g. muscle atrophy, joint ankylosis, pressure sores, calcinosis, aspiration pneumonia, visceral effects) but also those of treatment (Cushing syndrome, diabetes, osteoporosis, etc.) - determinant of prognosis and absent in the presented case. It provides thus life expectancy and quality close to those of the general population.

PL-7 antibodies presence could explain some of this case's particularities - mild and delayed onset myositis, the associated arthritis. As it also incurres interstitial lung fibrosis risk, pulmonology monitoring is required, stressing again the importance of multidisciplinary approach to these cases. Although the general rule is to treat a disease (and not some blood tests results), the presence of chemical (subclinical) rhabdomyolisis must determine a DMARD (Disease-Modifying anti Rheumatic Drug) introduction - methotrexate in our case - even before the appearance of clinical signs, in order to prevent loss of muscle mass and disease complications. Although absent in our case, the warning signs represented by dysphagia and dysphonia must be stressed again.

\section{CONCLUSION}

Early diagnosis, close monitoring and prompt treatment fine tuning to the level of disease activity ensure JDM adequate control and prevent disease and treatment complications, offering life expectancy and quality close to those of the general population.

Skin involvement may precede the muscular disease in JDM by months or years.

DMARDs may lack from the treatment of mild, skin-limited disease but must be promptly introduced in case of other forms.

Dysphonia and dysphagia are alarm signs which should determine an aggressive treatment (5), and also measures to protect the airway.

4. Clarissa Pilkington. Handbook of Systemic Autoimmune Diseases. Volume 6 Pediatrics in Systemic Autoimmune Diseases. Amsterdam, Elsevier, 2008

5. Thomas J.A. Lehman. A clinician's guide to rheumatic disease in children. New York, Oxford University Press, 2009
1. Lisa G. Rider, Carol B. Lindsley, James T. Cassidy. Textbook of pediatric rheumatology. Philadelphia, PA: Elsevier/ Saunders, 2005

2. Ronald M. Laxer, Susanne M. Benseler. Kelley's Textbook of Rheumatology. Philadelphia, PA: Elsevier/ Saunders, 2013

3. Denisa Predeteanu. Esentialul în reumatologie. Bucuresti, Ed. Amaltea, 2007 